\title{
Latest results from GlueX
}

\author{
Thomas Britton ${ }^{1, *}$ \\ for the GlueX Collaboration \\ ${ }^{1}$ Thomas Jefferson National Accelerator Facility Newport News, VA, USA
}

\begin{abstract}
The GlueX experiment is housed in the newest experimental hall at the Thomas Jefferson National Accelerator Facility in Newport News, Virginia. It was successfully commissioned in 2015 and is in its third year of data taking. GlueX uses a $12 \mathrm{GeV}$ electron beam incident on a diamond radiator, producing a linearly polarized, coherent Bremsstrahlung photon beam. The ultimate goal of GlueX is to search for exotic hybrid mesons (e.g. $q \bar{q} g$ ), with either exotic or conventional quantum numbers, whose existence, or lack thereof, would allow for the exploration of the gluon-gluon coupling present in QCD through the manifestation of hadrons with gluonic degrees of freedom. Photo-production at these energies is fairly unexplored and the linear beam polarization allows GlueX to discriminate between various production mechanisms which may be an effective way to identify such exotic hybrid mesons. In addition to exotic mesons, GlueX will also be poised to map out the conventional meson spectrum and to study the spectrum of excited vector mesons, which are often poorly understood. In these proceedings, we will present an overview of the GlueX experiment, its goals, current physics results, and future plans.
\end{abstract}

\section{Introduction}

The Thomas Jefferson National Accelerator Facility's (JLab) is home to the Continuous Electron Beam accelerator Facility (CEBAF) that has recently completed an upgrade from $6 \mathrm{GeV}$ to $12 \mathrm{GeV}$ and circulates electrons in a race track shaped accelerator. As part of the $12 \mathrm{GeV}$ upgrade program a new experimental hall, Hall-D, was constructed. The GlueX experiment is located after 5.5 passes through the accelerator in Hall-D. GlueX utilizes a photon beam produced by impinging the electron beam on a thin diamond radiator which produces a linearly polarized, coherent Bremsstrahlung photon beam with flux shown in Figure 1 a) and polarization measured via the Triplet Polarimeter (TPOL) to be upwards of $40 \%$ in region of the coherent peak (about 8.8 to $9.0 \mathrm{GeV}$ ) as seen in Figure 1. The photon beam is tagged, collimated, and passed into the experimental hall.

The GlueX spectrometer encases a liquid hydrogen target and provides good coverage for the detection of both neutral and charged final state particles. The barrel of the spectrometer is comprised primarily of a central drift chamber (CDC) and barrel calorimeter (BCAL) [2] in the radial direction and a set of drift chambers [3], a time-of-flight spectrometer and calorimeter in the forward direction (FDC, TOF, and FCAL respectively). The barrel detectors sit inside a two Tesla superconducting magnet. The entire GlueX detector setup can

\footnotetext{
*e-mail: tbritton@jlab.org
} 
be seen in Figure 2. All together, GlueX has a beam resolution of about $0.1 \%$ and momentum/energy resolutions of between 1 and $5 \%$.
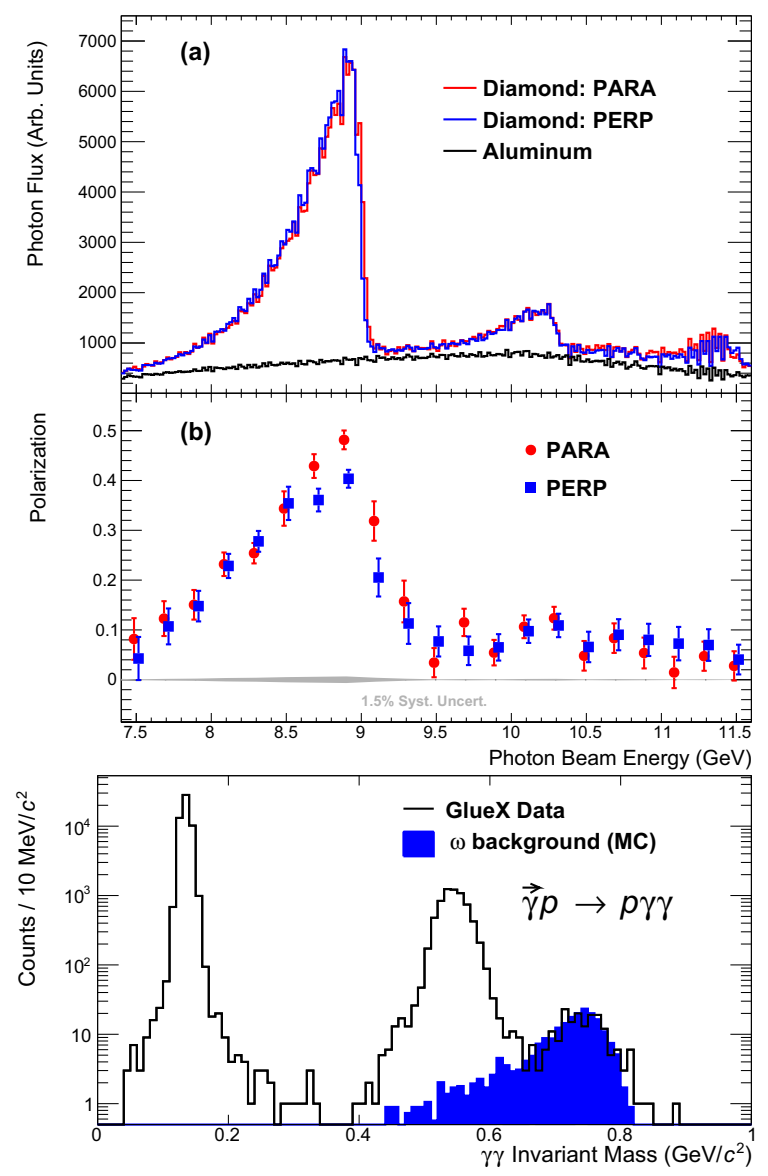

Figure 1: (Top) a) The photon beam flux for two beam polarization angles ("PARA" in red and "PERP" blue). b) The polarization of the beam for two polarization angles "PARA" (red) "PERP" (blue) as measured by the Triplet Polarimeter (TPOL) [1]. (Bottom) The $\gamma \gamma$ invariant mass showing clean $\pi^{0}$ and $\eta$ samples with the $\omega$ contamination of the $\eta$ sample, as estimated by Monte Carlo simulations, in blue.

\section{Purpose}

The quark model of Quantum Chromodynamics (QCD) allows for colorless groupings of quarks such as mesons $(q \bar{q})$, baryons $(q q q)$, tetra-quarks $(q \bar{q} q \bar{q})$, and penta-quarks $(q q q q \bar{q})$ [4]. But these are not the only states that are predicted. Lattice QCD (LQCD) also predicts a spectrum of bound states beyond those in the constituent quark model [5]; these are the socalled glueballs, which are comprised of only gluons $(g g)$, and hybrid mesons $(q \bar{q} g)$. GlueX is designed to hunt for signals from these "exotics". The non-relativistic quark model relates total spin $(\mathrm{J})$, parity $(\mathrm{P})$, and charge conjugation $(\mathrm{C})$ to the spin $(\mathrm{S})$ and total angular momentum (L) by the following equations: $J=L+S, P=-1^{L+1}$, and $C=-1^{L+S}$. These relationships 


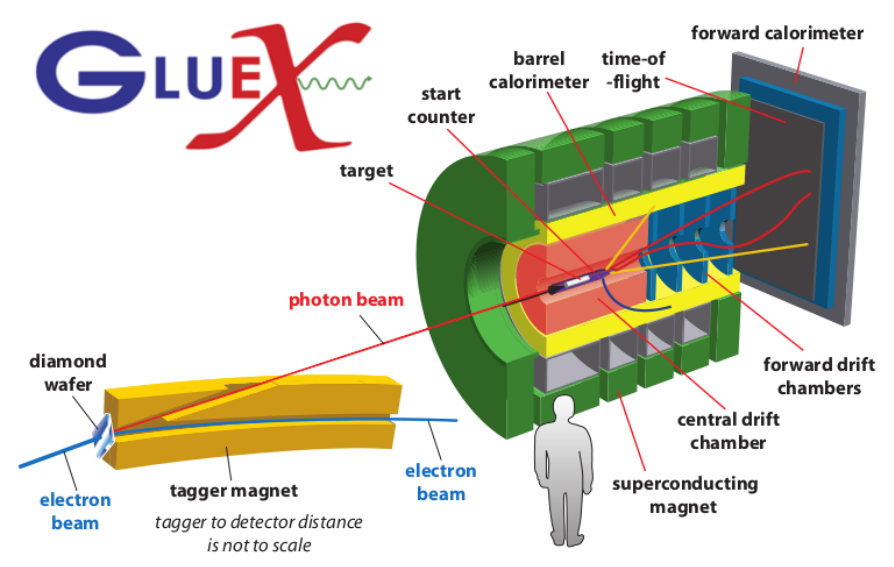

Figure 2: Shown is the layout of the GlueX experiment with person for scale.

restrict the possible values of $J^{P C}$, forbidding $J^{P C}$ values of $0^{+-}, 1^{-+}, 2^{+-}$, and so on. Thus observations of states with these "forbidden" or "exotic" quantum numbers would provide direct evidence for states beyond the constituent quark model. Even with a rich spectrum predicted most experimental searches were limited to the $1^{-+}$isovector state, often referred to as $\pi_{1}(1600)$ state. For example Compass noted an enhancement in the $1^{-+}$wave in the $\eta^{\prime} \pi^{-}$ system in the reaction $\pi^{-} p \rightarrow \pi^{-} \eta^{\prime} p$ [6].

GlueX is in a unique position to search for signs of exotics, including hybrid mesons. Firstly, there are very few photo-production experiments that have searched in the energy ranges that GlueX is searching (roughly $3 \mathrm{GeV}$ to $12 \mathrm{GeV}$ ). This beam energy range exactly corresponds to the mass range where we expect such hybrids to be found. Secondly, GlueX uses a linearly polarized photon beam. This enables GlueX to probe production mechanisms in greater detail. Finally, photons couple via vector meson dominance to a wide variety of states, including those with exotic $J^{P C}$ as shown in Table 1.

Table 1: A set of possible exchanges in $\gamma p$ interactions and their associated exotic final states.

\begin{tabular}{ll|ll}
\hline Exchange & & Exotic Final States \\
\hline $\mathbb{P}$ & $0^{++}$ & $b, h, h^{\prime}$ & $2^{+-}, 0^{+-}$ \\
$\pi^{0}$ & $0^{-+}$ & $b_{2}, h_{2}, h_{2}^{\prime}$ & $2^{+-}$ \\
$\pi^{ \pm}$ & $0^{-+}$ & $\pi_{1}^{ \pm}$ & $1^{-+}$ \\
$\omega$ & $1^{--}$ & $\pi_{1}, \eta_{1}, \eta_{1}^{\prime}$ & $1^{-+}$
\end{tabular}

\section{GlueX analyses}

Thus far GlueX has collected over 200 billion triggers in approximately 100 days worth of data collection. Of this data approximately 25 percent has been analyzed and already GlueX is producing various physics results. 

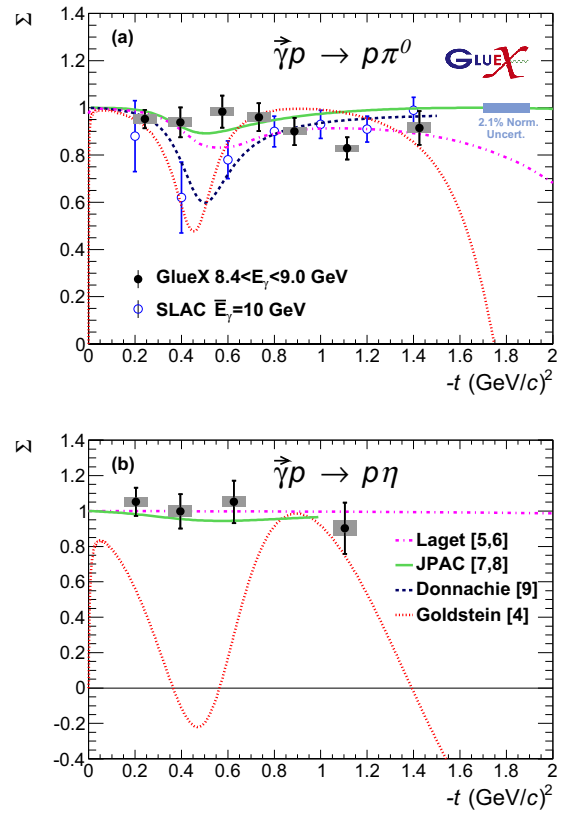
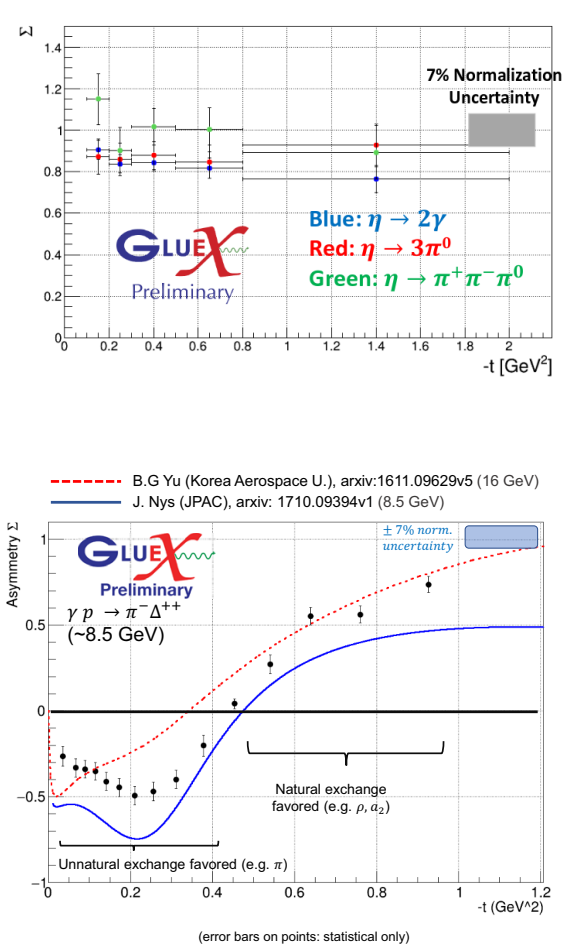

(error bars on points: statistical only)

Figure 3: (Left) The beam asymmetry measurements of $\pi^{0}$ along with prior measurements and theory curves (a) and $\eta$ with theory curves (b). (Right-Top) The preliminary results of the updated analysis of the $\eta$ beam asymmetry using roughly five times the statistics [7]. (RightBottom) The preliminary results of the beam asymmetry of $\pi^{-}$from the reaction $\gamma p \rightarrow \pi^{-} \Delta^{++}$ [8].

\subsection{Beam asymmetries}

Linearly-polarized beam asymmetries $(\Sigma)$ provide insights into the production mechanisms involved and are the first stop of the analysis road-map. A measurement of the beam asymmetry of $\pi^{0}$ in $\gamma p \rightarrow \pi^{0} p$ was among the first physics results from GlueX. This analysis was easily extended to $\eta$ in $\gamma p \rightarrow \pi^{0} p$ where there were no previous measurements and together formed the first GlueX publication [9].

The analysis was performed on relatively clean samples of $\pi^{0}$ and $\eta$ (Figure 1). In the case of $\pi^{0}$ (Figure 3 a) the dip observed by SLAC at $-t \approx 0.4 \mathrm{GeV} / \mathrm{c}^{2}$ and modeled by Laget, Donnachie and Goldstein is not seen. There is better agreement between the predictions of the Joint Physics Analysis Center (JPAC) and the GlueX data. Additionally, the value of $\Sigma \approx 1$ indicates vector exchange dominance. Looking at the beam asymmetry of $\eta$ GlueX finds a similar picture; with $\Sigma \approx 1$, indicating vector meson dominance and agreeing with JPAC predictions.

An update to the $\eta$ beam asymmetry, involving roughly five times the initially published data set, is underway. Preliminary results shows good agreement between three decay modes of $\eta: \eta \rightarrow \gamma \gamma, \eta \rightarrow \pi^{0} \pi^{0} \pi^{0}$, and $\eta \rightarrow \pi^{+} \pi^{-} \pi^{0}$ (Figure 3 ). The analysis of neutral pseudoscalars continues with the analysis of the beam asymmetry of $\eta^{\prime}$. In all of these cases, prelim- 

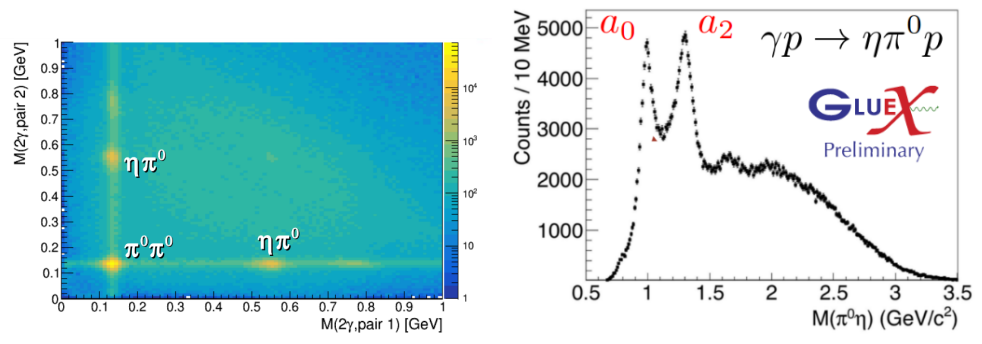

Figure 4: (Left) The two-dimensional plot of the invariant mass of one set of $\gamma \gamma$ versus the other set of $\gamma \gamma$ from $\gamma p \rightarrow \gamma \gamma \gamma \gamma p$. Clearly visible are signals for both $\eta \pi^{0}$ and $\pi^{0} \pi^{0}$. (Right) The invariant mass of $\pi^{0} \eta$ showing a prominent $a_{0}(980)$ and $a_{2}(1320)$.
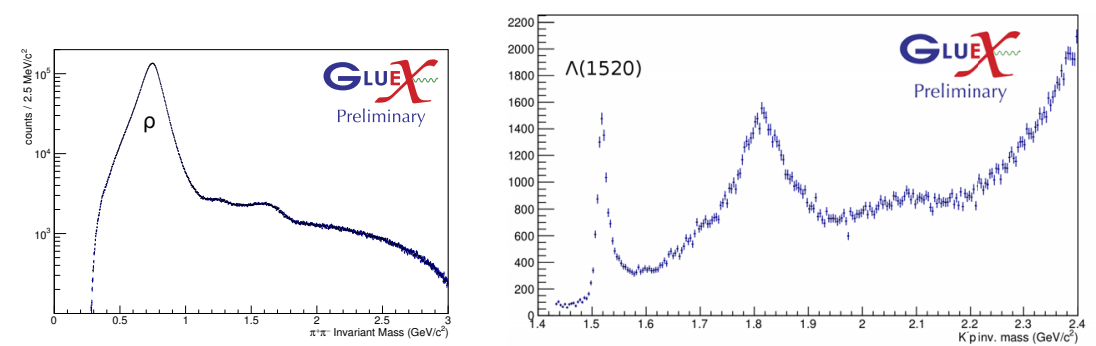

Figure 5: (Left) The invariant mass of $\pi^{+} \pi^{-}$with about 100 times the statistics of the earlier measurement by SLAC. (Right) The invariant mass of $K^{-} p$ showing a multitude of features beyond $\Lambda(1520)[10]$.

inary results of GlueX indicate $\Sigma$ of approximately one across the measured $t$ range, implying vector exchange dominance.

Charged pseudo-scalars such as $\pi^{-}$have also been analyzed in the reaction $\gamma p \rightarrow \pi^{-} \Delta^{++}$. Preliminary results of the beam asymmetry of $\pi^{-}$(Figure 3 ) indicate a more complex $t-$ dependence with lower $|t|$ indicating a preference for unnatural exchange (e.g pion) and higher $|t|$ preferring more natural exchange (e.g. $\rho, a_{2}$ ).

\subsection{Spectroscopy}

GlueX has good acceptance for a plethora of final states which enables opportunities in spectroscopy. The data from prior experiments is often sparse in channels with multiple neutral states and GlueX already has much more data than prior experiments. For example, GlueX finds clear signals for $\pi^{0} \pi^{0}$ and $\eta \pi^{0}$ production (Figure 4) in the reaction $\gamma p \rightarrow \gamma \gamma \gamma \gamma p$. The invariant mass of $\pi^{0} \eta$ reveals prominent $a_{0}$ and $a_{2}$ signals [11] (Figure 4). The richness of features present in the GlueX $\eta \pi^{0}$ spectrum is in direct contrast to some earlier measurements, for example, those from E852 [12]. Continuing neutral spectroscopy, GlueX is also able to reconstruct five photon final states and observes $b_{1}(1235)$ in its dominant decay mode $\gamma p \rightarrow b_{1}(1235) p, b_{1}(1235) \rightarrow \omega \pi^{0}, \omega \rightarrow \pi^{0} \gamma$

Leaving neutral states GlueX can also study the spectrum of charged final states. In $\gamma p \rightarrow \pi^{+} \pi^{-} p$ the singular feature in the invariant mass of $\pi^{+} \pi^{-}$at about $1.5 \mathrm{GeV}$ seen by SLAC [13] is resolved with approximately one hundred times the data, into the $f_{2}(1270)$ 

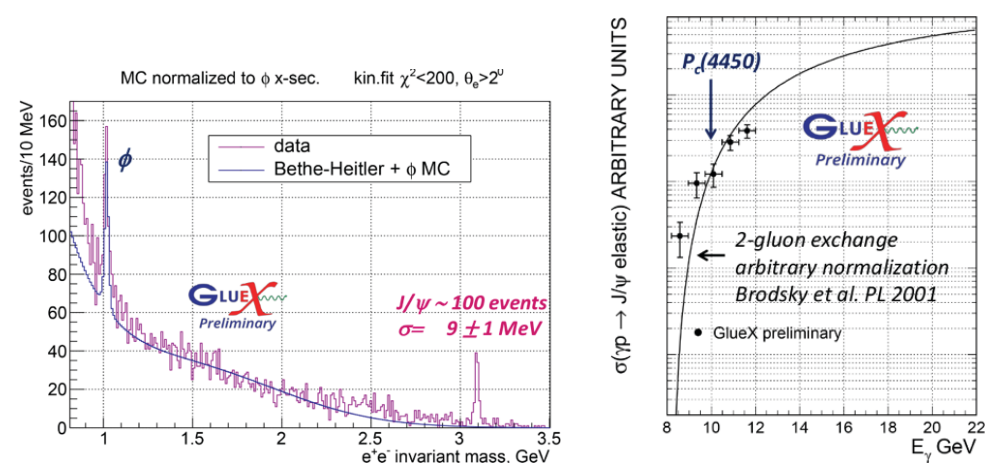

Figure 6: (Left) The invariant mass of $e^{+} e^{-}$showing a clear signal at the $J / \psi$ mass, using only a small subset of the data collected by GlueX. (Right) The elastic cross-section of $\gamma p \rightarrow J / \psi$ in arbitrary units.

and another feature, possibly $\rho^{\prime}$, at approximately $1.6 \mathrm{GeV}$ (Figure 5). Further analysis will be needed to discern the true nature of these enhancements. In the reaction $\gamma p \rightarrow K^{+} K^{-} p$ the clear signal of $\phi$ is present in its dominant decay mode. The invariant mass of $K^{-} p$ reveals many interesting features which could be associated with higher-mass lambdas (above $\Lambda(1520)$ ) in the reaction $\gamma p \rightarrow K^{+} \Lambda$, with $\Lambda$ decaying to $K^{-} p$ (Figure 5). Many of the higher mass $\Lambda$ states beyond $\Lambda(1520)$ have poorly measured masses and width. With proper analysis the GlueX data may be able to resolve some of the uncertainties with these higher mass features.

\section{$3.3 \mathrm{~J} / \psi$ photo-production}

The discovery, by LHCb, of pentaquark candidates in $J / \psi p$ from $\Lambda_{b}$ decays [14] has spurred a great deal of interest in the $J / \psi p$ system. These pentaquark candidates should be accessible to GlueX and are expected to appear as s-channel resonances at photon energies of approximately $10 \mathrm{GeV}[15,16]$. Studying $J / \psi$ near threshold can give information as to the nucleon distribution and any signals in $\gamma p \rightarrow J / \psi p$ would provide important confirmation of the states observed by LHCb. GlueX should be able to measure the cross-section and can measure the branching ratio, or set limits, of $P_{c} \rightarrow J / \psi p$. Even with a small fraction of the data collected by GlueX there is a clear signal for $J / \psi$ (Figure 6) and with that signal, proof that measurements related to the LHCb pentaquark states will be able to be performed by GlueX. Preliminary results are starting to take shape and should fill in the gaps in prior measurements (Figure 6).

\section{GlueX future}

In the near-term GlueX will install and commission a DIRC (Detection of Internally Reflected Cherenkov light) detector that should greatly enhance the ability of GlueX to distinguish kaons from pions at momentums almost twice as high as current time-of-flight measurements can provide [17]. With partial installation and commissioning occurring in 2018 GlueX will have a greatly enhanced strangeness program enabling searches for signals from exotic candidates in more channels. 


\section{Summary}

GlueX has completed roughly three years of data taking since 2015. Already physics results are taking shape with a multitude of beam asymmetry measurements. Some of these results represent first measurements at these energies (e.g $\eta$ ) and others show some degree of disagreement with prior measurements $\left(\mathrm{e} . \mathrm{g} \pi^{0}\right)$. The GlueX spectrometer has been performing well allowing the reconstruction of reactions with multiple neutral final states (e.g. $4 \gamma$ and $5 \gamma$ final states) where often the prior data is sparse and statistics are dwarfed by GlueX. Since the discovery of pentaquark candidates by $\mathrm{LHCb}$ in $J / \psi p$ has prompted a lot of interest in measurements of $J / \psi$ in photoproduction on proton targets. There is an expectation that these pentaquark candidates should reveal themselves as s-channel resonances with photon energies of about $10 \mathrm{GeV}$. This puts GlueX in prime position to measure or set limits on the pentaquark candidates of LHCb. Additionally, a DIRC detector will be partially installed and commissioned in 2018 and should provide much improved $\pi / K$ separation.

\section{Acknowledgements}

This material is based upon work supported by the U.S. Department of Energy, Office of Science, Office of Nuclear Physics under contract DE-AC05-06OR23177.

\section{References}

[1] M. Dugger et al., NIM in Physics Research 867, 115 (2017)

[2] T.D. Beattie et al., NIM in Physics Research 896, 24 (2018)

[3] L. Pentchev et al., NIM in Physics Research 845, 281 (2017)

[4] M. Gell-Mann, Physics Letters 8, 214 (1964)

[5] Jozef J. Dudek et al., Physical Review D 88, 094505 (2013)

[6] C. Adolf et al., Physics Letters B 740, 303 (2015)

[7] Will McGinley, in these proceedings

[8] John Zarling, in these proceedings

[9] H. Al Ghoul et al., Physical Review C 95, 042201(R) (2017)

[10] Peter Pauli, in these proceedings

[11] Shuang Han, in these proceedings

[12] G. S. Adams et al., Physics Letters B 657, 27 (2007)

[13] K. Abe et al., Physical Review Letters 53, 751 (1984)

[14] R. Aaij et al., Physical Review Letters 115, 072001 (2015)

[15] V. Kubarovsky and M. B. Voloshin, Physical Review D 92, 031502(R) (2015)

[16] Marek Karliner, Jonathan L. Rosner, arXiv:1508.01496v3 (2015)

[17] J. Stevens et al., JINST 11(07), C07010 (2016) 